\title{
MAXIMAL SPEED OF QUANTUM PROPAGATION
}

\author{
J. ARBUNICH, F. PUSATERI, I. M. SIGAL, AND A. SOFFER
}

\begin{abstract}
For Schrödinger equations with both time-independent and timedependent Kato potentials, we give a simple proof of the maximal speed bound. The latter states that the probability to find the quantum system outside a ball of radius proportional to the time lapsed decays as an inverse power of time. We give an explicit expression for the constant of proportionality in terms of the maximal energy available to the initial condition. For the time-independent part of the interaction, we require neither decay at infinity nor smoothness.

Key words: Schrödinger equation, quantum dynamics, propagation speed, light cone, propagation estimates, quantum information, quantum scattering.
\end{abstract}

\section{INTRODUCTION}

It was shown in [19] that there is a constant $c$ such that the probability to find a quantum mechanical system outside the ball of radius $c t$ decays as an inverse power of time $t$. We call this result the maximal propagation speed bound. It complements the minimal propagation speed estimates obtained in [19, 21, 14]; see also [6], [11] for related results, and [13] for a review of the latter.

In this paper, we give a simple proof of the maximal propagation speed (MPS) bound for Schrödinger (or von Neumann) equations with time-independent and time-dependent potentials. We also give an upper bound on the infimum of such constants $c$ as above, which we call the maximal propagation speed, in terms of the maximal energy of the state involved. Our assumption on the time-independent part of the interaction is rather general and allows e.g. many-body potentials with Coulomb singularities. In particular, neither decay at infinity nor smoothness is required.

There is a certain parallel between the MPS bound and the Lieb-Robinson one, which has found many applications and has been discussed extensively in the literature, see [17, 9, 16, 5] for a review and some recent papers. Both deal with the maximal propagation speed of information in quantum systems over large distances. The seeming discrepancy in that the Lieb-Robinson bounds do not depend on the energy is due to the fact that, with exception of [9], these bounds are obtained for discrete Hamiltonians for which the energy is bounded to begin with. ([9] uses an explicit momentum cut-off in the solution.)

Date: March 3, 2021. 
The paper is organized as follows. In Section 2 we formulate the problem precisely and state our first main result dealing with time-independent potentials. In Section 3 we state the corresponding result for time-dependent potentials. In Sections 4 and 5, we prove the corresponding results and in Appendix $\mathrm{A}$ we collect commutator estimates (non-Abelian functional calculus) based on those described in [13].

In what follows, the relation $A \lesssim B$ means that there is a constant $C>0$ independent of the parameters $s, t, a, b, c$ appearing below and s.t. $A \leq C B$.

\section{The PROBLEM AND RESUlts FOR TIME-INDEPENDENT POTENTIALS}

We consider the Schrödinger equation $i \partial_{t} \psi_{t}=H \psi_{t}$, with a Schrödinger operator $H=-\frac{1}{2} \Delta+V(x)$ on $L^{2}\left(\mathbb{R}^{d}\right)$ and an initial condition $\psi$. We will assume that $V(x)$ is $\Delta$-bounded with the relative bound $<1$, i.e. it satisfies (with $\|\cdot\|$ being the norm in $\left.L^{2}\left(\mathbb{R}^{d}\right)\right)$

$$
\exists 0 \leq a<1, b>0: \quad\|V u\| \leq a\left\|\frac{1}{2} \Delta u\right\|+b\|u\| .
$$

Then by Kato's result (see e.g. [2]), $H$ is self-adjoint on the domain of $\Delta$.

We say that a state $\psi$ (or $\psi_{t}=e^{-i H t} \psi$ ) obeys the maximal propagation speed (MPS) bound if there is a constant $c<\infty$ s.t., as $|t| \rightarrow \infty$,

$$
\int_{|x| \geq c|t|} d x\left|\psi_{t}(x)\right|^{2} \rightarrow 0 .
$$

Moreover, the scalar $c_{\max }:=\inf \{c:(\underline{2.2})$ holds $\}$ will be called the maximal propagation speed.

Let $\chi_{A}$ stand for the characteristic function of a set $A$. In this paper, we show that any state $\psi \in \operatorname{Ran} \chi_{I}(H)$ obeys the MPS bound and the maximal propagation speed $c_{\max }$ is bounded above by the constant

$$
k:=\left\||p| \chi_{I}(H)\right\|
$$

where $p:=-i \nabla$ and $I$ is an interval in $\mathbb{R}$. Note that, if $I:=(-\infty, E]$ for some energy $E>0$, then due to (2.1),$k^{2} \leq 2(E+b) /(1-a)$. We have

Theorem 2.1. [Maximal propagation speed bound] Suppose that $H$ satisfies (2.1). Let $g \in C_{0}^{\infty}(I)$ be real and let $A_{\rho}^{ \pm}:=\left\{x \in \mathbb{R}^{d}: \pm|x| \geq \pm \rho\right\}$. If $c>k$, then

$$
\left\|\chi_{A_{c t+a}^{+}} e^{-i H t} g(H) \chi_{A_{b}^{-}}\right\| \lesssim t^{-n}
$$

for $t>1$ and any $n$, uniformly in a in any region $0<b<a \lesssim t$.

Earlier results. MPS bounds were first given in [19] and then extended in [21] and [1] and used in scattering theory in [20, 18, 10, 3, 7].

The main contributions of this paper compared to earlier work are: 
(a) a significant extension of the class of potentials (in particular, no smoothness, or decay at infinity is required);

(b) a much simpler proof;

(c) the determination of the precise maximal velocity bound (see (2.3) ).

Light cone. Inequality (2.4) (see also (2.6) ) implies that the probability to find the quantum system outside the ball $\langle x\rangle \leq c t+a$ decays with time. Moreover, for well localized initial conditions, it gives a rate of decay.

Below, we prove a stronger result implying a limitation on the speed of propagation of information. Let $\alpha_{t}$ be the Heisenberg evolution, $\alpha_{t}(B):=e^{i H t} B e^{-i H t}$. Then we show for $c>k, \rho$ satisfying $\rho>a+c t$, for $a$ sufficiently large,

$$
\left\|\chi_{A_{\rho}^{+}} \alpha_{t}\left(\chi_{A_{b}^{-}} g(H)\right)\right\| \lesssim \rho^{-n}
$$

i.e. it takes at least $\rho / c$ units of time for a signal originating in $A_{b}^{-}$to reach $A_{\rho}^{+}$.

Density matrices. Since Theorem 2.1 and its version in (2.5) deal with propagation of observables, they are valid also for states described by density operators, i.e. for the von Neumann dynamics.

Weighted estimates. Inequality (2.4) implies weighted estimates. Indeed, using

$$
\langle x\rangle^{-\alpha}=\langle x\rangle^{-\alpha} \chi_{A_{\epsilon t / 2}^{-}}+O\left(t^{-\alpha}\right)
$$

and using (2.4), with $b=\epsilon t / 2$, we find, for $n \geq \alpha$,

$$
\left\|\chi_{A_{(c+\epsilon) t}^{+}} e^{-i H t} g(H)\langle x\rangle^{-\alpha}\right\| \lesssim t^{-\alpha}
$$

Abstract formulation. Theorem 2.1 could be stated in an abstract setting for a pair of self-adjoint operators $H$ and $X$ with multiple commutators required to be $H$-bounded, with some extra care in the definition of the commutators for unbounded operators. Here we deal with Schrödinger operators $H=\frac{1}{2} p^{2}+V(x)$ and with the particle position coordinate $x$, more precisely, with $\langle x\rangle$.

Time reversal. The results for $t<0$ are obtained by complex conjugation $\mathcal{C}$ : $\psi \rightarrow \bar{\psi}$, using $\mathcal{C} H \mathcal{C}=H, \mathcal{C} x \mathcal{C}=x$ : (2.4) transform into

$$
\left\|\chi_{A_{c t+a}^{+}} e^{i H t} g(H) \chi_{A_{b}^{-}}\right\| \lesssim t^{-n}
$$

for $t>1$ and for any $n$, which can be reformulated in terms of $t<-1$.

Approach. Our approach can be thought of as microlocal analysis with an operator functional calculus replacing the pseudodifferential one. It is based on the method of propagation observables which we now explain.

We consider a time-dependent, non-negative operator-family (propagation observable) $\Phi_{t}$. We would like to obtain propagation estimates of the form $\left\|\Phi_{t} \psi_{t}\right\| \lesssim t^{-m}$. 
Denote the inner product in $L^{2}\left(\mathbb{R}^{d}\right)$ by $\langle\cdot, \cdot\rangle$, so that $\|\cdot\|=\sqrt{\langle\cdot, \cdot\rangle}$ and let $\psi_{t}=e^{-i H t} g(H) \phi$ be a spectrally localized solution to the Schrödinger equation and let $\langle A\rangle_{t}:=\left\langle\psi_{t}, A \psi_{t}\right\rangle$. Note the relation

$$
\frac{d}{d t}\left\langle\Phi_{t}\right\rangle_{t}=\left\langle D \Phi_{t}\right\rangle_{t} ; \quad D \Phi_{t}=i\left[H, \Phi_{t}\right]+\frac{\partial}{\partial t} \Phi_{t} .
$$

We call $D$ the Heisenberg derivative. Using $\left\langle\Phi_{t}\right\rangle_{t}=\left\langle\Phi_{0}\right\rangle_{0}+\int_{0}^{t} \partial_{r}\left\langle\Phi_{r}\right\rangle_{r} d r$, we find

$$
\left\langle\Phi_{t}\right\rangle_{t}-\int_{0}^{t}\left\langle D \Phi_{r}\right\rangle_{r} d r=\left\langle\Phi_{0}\right\rangle_{0},
$$

which we call the basic equality. If $g(H) D \Phi_{t} g(H) \leq 0$, modulo fast time-decaying terms, then, after pulling $g(H)$ out of $\psi_{t}$, this relation gives estimates on the positive terms $\left\langle\Phi_{t}\right\rangle_{t}$ and $-\int_{0}^{t}\left\langle D \Phi_{r}\right\rangle_{r} d r$.

We will consider propagation observables of the form $\Phi_{t s}=f\left(x_{t s}\right)$, where $x_{t s}:=$ $s^{-1}(\langle x\rangle-a-c t)$, with $s \geq t$, and $f$ is a non-negative, non-decreasing function supported in $(0, \infty)$. The factor $s^{-1}$ is introduced to control multiple commutators and commutator products. It can be thought of as an adiabatic or semi-classical parameter.

The energy cut-off $g(H)$ appearing next to $D \Phi_{t}$ is pulled out of $\psi_{0}$, by commuting it with the evolution $e^{-i H t}$. The latter simple but crucial fact expresses the conservation of energy for the Schrödinger equation. It is not valid for Schrödinger equations with time-dependent Schrödinger operators, $H(t)$. This is the main obstacle in treating such equations. We overcome it by introducing the asymptotic energy cut-offs and proving the pull-through relation, replacing the commutativity of $g(H)$ and $e^{-i H t}$. In what follows, we let $g \in C_{0}^{\infty}(I)$.

\section{Time-DEPEndent Potentials}

Consider time-dependent hamiltonians of the form

$$
H_{t}:=H+W_{t},
$$

where $H$ is as above and $W=W(x, t)$ is a real, time-dependent potential satisfying

$$
\partial_{x}^{\alpha} W_{t}(x)=O\left(\langle t\rangle^{-\mu-|\alpha|}\right), \quad \mu>1,|\alpha| \leq 2 .
$$

Here $\langle t\rangle:=\left(1+t^{2}\right)^{1 / 2}$, but in what follows we consider only $t>0$ and ignoring the singularity at $t=0$, we write $t$ for $\langle t\rangle$.

Let $U_{t}:=U(t, 0)$ be the evolution generated by $H_{t}$. Here the crucial role is played by the asymptotic energy cut-offs introduced in [19]:

Proposition 3.1. [Asymptotic energy cut-off] Let condition (3.2) hold but with $\mu>0$ instead of $\mu>1$. Then the following operator-norm limit exists

$$
g_{+}(H):=\lim _{t \rightarrow \infty} U_{t}^{-1} g(H) U_{t} .
$$


Proof. Define $g_{t}(H):=U_{t}^{-1} g(H) U_{t}$, and write $g_{t}(H)$ as the integral of the derivative and use that $\partial_{r} g_{r}(H)=i U_{r}^{-1}\left[g(H), W_{r}\right] U_{r}$ to obtain

$$
g_{t}(H)=g(H)+i \int_{0}^{t} U_{r}^{-1}\left[g(H), W_{r}\right] U_{r} d r .
$$

Since $\left[g(H), W_{r}\right]=O\left(r^{-\mu-1}\right)$ (see Lemma A.2 of Appendix A), this shows that $g_{+}(H)=\lim g_{t}(H)$ (the operator norm limit) exists provided $\mu>0$.

Remark. As was noticed by one of the referees, (a) assumption (3.2) on the timedependent potential $W_{t}$ can be relaxed, e.g. to $\int_{0}^{\infty}\left\|\partial_{x}^{\alpha} W_{t}(x)\right\| d t<\infty,|\alpha| \leq 2$; (b) for $\mu>1$ (which is used in our proofs below) the proof of Proposition 3.1 is simpler and the obvious estimate $\left\|\left[g(H), W_{r}\right]\right\| \leq 2\|g(H)\|\left\|W_{r}\right\|=O\left(r^{-\mu}\right)$ is sufficient. So, we could have assumed e.g. $\left\|W_{t}(H+1)^{-1}\right\|_{L^{\infty}} \lesssim\langle t\rangle^{-\mu}, \mu>1$.

Eq. (3.4) implies that

$$
g_{+}(H)-g_{t}(H)=i \int_{t}^{\infty} U_{r}^{-1}\left[g(H), W_{r}\right] U_{r} d r=O\left(t^{-\mu}\right),
$$

which, together with $U_{t} g_{t}(H)=U_{t} U_{t}^{-1} g(H) U_{t}=g(H) U_{t}$, implies

Corollary 3.2. [Pull-through relation] With the notation and assumptions above, see (3.1), (3.2) and (3.3), we have

$$
U_{t} g_{+}(H)=g(H) U_{t}+O\left(t^{-\mu}\right) \text {. }
$$

Using the above key relation with $\mu>1$,and additional commutator estimates, we are able to extend Theorem 2.1 to time-dependent potentials:

Theorem 3.3. [Maximal propagation speed for $t$-dependent potentials] Suppose that $H_{t}$ is of the form (3.1), with $H$ and $W_{t}$ satisfying (2.1) and (3.2). Let $g \in C_{0}^{\infty}(I)$ be real. If $c>k$, then

$$
\left\|\chi_{A_{c t+a}^{+}} U_{t} g_{+}(H) \chi_{A_{b}^{-}}\right\| \lesssim t^{-1 / 2}
$$

for $t>1$, uniformly in $a$ in any region $0<b<a \lesssim t$. Here $A_{\rho}^{ \pm}$are the sets defined in Theorem 2.1.

The next result from [19] shows that the initial conditions of the form $g_{+}(H) \chi_{A_{b}^{-}} \phi$, for various $g(H), b>0$ and $\phi \in L^{2}$, form a dense set.

Theorem 3.4. Let $\left(I_{n}\right) \subset \mathbb{R}$ be a sequence of increasing intervals with $\lim _{n \rightarrow \infty} I_{n}=$ $\mathbb{R}$, and let $g_{n} \in C_{0}^{\infty}(\mathbb{R})$ be such that $g_{n} \equiv 1$ on $I_{n}$. Let $g_{n,+}:=\lim U_{t}^{-1} g_{n}(H) U_{t}$, as in (3.3). Then

$$
s-\lim _{n \rightarrow \infty} g_{n,+}(H)=\mathrm{id} .
$$

See [19] for the proof of this theorem.

As was mentioned after (2.9), to bound $D \Phi_{t s}$ from above, we have 'to pull $g(H)$ from $\psi_{0}$ ' through $U_{t}$. For the time-dependent situation this is achieved using (3.6). 
Note that we need $\mu>1$ for the remainder term in (3.6) to lead to an integrable contribution.

\section{Proof of Theorem 2.1}

Definitions and notation. We fix $c>v$ and let $\mathcal{F}$ be the set of functions $0 \leq f \in$ $C^{\infty}(\mathbb{R})$, supported in $\mathbb{R}^{+}$and satisfying $f(\lambda)=1$ for $\lambda \geq c-v$, and $f^{\prime} \geq 0$, with $\sqrt{f^{\prime}} \in C^{n}$. Here and in the rest of the section, $n$ is the same as in (2.4).

We say a function $h$ is admissible if it is smooth, non-negative with $\operatorname{supp} h \subset$ $(0, c-v)$ and $\sqrt{h} \in C^{n}$. Note that if $h$ is admissible, then $h=f^{\prime}$, with

$$
f(\lambda)=\int_{-\infty}^{\lambda} h(s) d s \text { and }\left(\int_{-\infty}^{\infty} h(s) d s\right)^{-1} f \in \mathcal{F} .
$$

Moreover, we will use the following notation (for any $\phi \in L^{2},\|\phi\|=1$ ):

$$
\chi_{b}^{-}:=\chi_{A_{b}^{-}}, \quad \psi_{t}:=e^{-i H t} g(H) \chi_{b}^{-} \phi \quad \text { and } \quad x_{t s}:=s^{-1}(\langle x\rangle-a-v t)
$$

and the convention that $A \dot{\leq} B$ and $A \lesssim B$ mean that for any $n>0$, there is $C>0$ s.t. $A \leq B+C s^{-n}$ and $A \lesssim B+C s^{-n}$, respectively.

We begin the proof of Theorem 2.1 with the velocity bound below.

Lemma 4.1. Given $f \in \mathcal{F}$, let $u^{2}=f^{\prime}$. Then, for any $n$, there is $\tilde{u}$, with $\tilde{u}^{2}$ admissible, s.t.

$$
\left\|p u\left(x_{t s}\right) g(H) \psi\right\| \dot{\leq} k\left\|u\left(x_{t s}\right) g(H) \psi\right\|+s^{-1}\left\|\tilde{u}\left(x_{t s}\right) g(H) \psi\right\| .
$$

Proof. Writing $g(H)=\tilde{g}(H) g(H)$, with $\tilde{g} \in C_{0}^{\infty}(I)$, with $\tilde{g}=1$ on $\operatorname{supp} g$, and commuting $\tilde{g}(H)$ to the left, we find

$$
p u\left(x_{t s}\right) g(H)=p \tilde{g}(H) u\left(x_{t s}\right) g(H)+p\left[u\left(x_{t s}\right), \tilde{g}(H)\right] g(H) .
$$

Now, by (A.3),$p\left[u\left(x_{t s}\right), \tilde{g}(H)\right]=\sum_{k=1}^{n-1} \frac{s^{-k}}{k !} p B_{k} u^{(k)}\left(x_{t s}\right)+O\left(s^{-n}\right)$ for any $n$, with $p B_{k}$ bounded. With this, taking the norm of (4.2) and using $\|p \tilde{g}(H)\| \leq k$, we arrive at (4.1) with $\tilde{u}^{2}:=C\left(u^{2}+\sum_{k=1}^{n-1} s^{-k}\left(u^{(k)}\right)^{2}\right)$, for some $C>0$.

Next is a key statement in the proof of Theorem 2.1;

Proposition 4.2. Under the hypothesis of Theorem 2.1, for any $f \in \mathcal{F}$ and any $n$, there is $\tilde{f} \in \mathcal{F}$ s.t., for $s \geq t$,

$$
\int_{0}^{t}\left\langle f^{\prime}\left(x_{r s}\right)\right\rangle_{r} d r \lesssim s\left\langle\tilde{f}^{\prime}\left(x_{0 s}\right)\right\rangle_{0}+O\left(s^{-n}\right)
$$

Proof. We proceed inductively, showing that (4.3) holds for $n=0$ and then assuming it holds for some $n=n^{\prime} \in \mathbb{R}$ and proving that it holds for $n=n^{\prime}+1$, and proceed in this way until we reach an arbitrary $n$. To this end, we use the time dependent observable

$$
\Phi_{s}(t)=f\left(x_{t s}\right), \quad f \in \mathcal{F},
$$


with $0 \leq t \leq s$. In order to estimate $\left\langle\Phi_{s}(t)\right\rangle_{t}=\left\langle\psi_{t}, \Phi_{s}(t) \psi_{t}\right\rangle$, we apply (2.8) and the basic equality (2.9) . We start by computing $D \Phi_{s}(t)$. First, we have

$$
\frac{\partial}{\partial t} \Phi_{s}(t)=-s^{-1} v f^{\prime}\left(x_{t s}\right)
$$

Then, we let $\gamma:=\frac{1}{2}(p \cdot \nabla\langle x\rangle+\nabla\langle x\rangle \cdot p)$, with $p:=-i \nabla$. Factorizing $f^{\prime}=u^{2}$ and using that $[H,\langle x\rangle]=\gamma$ and $[[\gamma, u], u]=0$, we find

$$
\begin{aligned}
i\left[H, \Phi_{s}(t)\right]= & \frac{i}{2}\left[p^{2}, \Phi_{s}(t)\right]=\frac{1}{2} s^{-1}\left(\gamma f^{\prime}\left(x_{t s}\right)+f^{\prime}\left(x_{t s}\right) \gamma\right) \\
& =s^{-1} u\left(x_{t s}\right) \gamma u\left(x_{t s}\right) .
\end{aligned}
$$

This equation, together with Eq. (4.5), yields:

$$
D \Phi_{s}(t)=s^{-1} u\left(x_{t s}\right)(\gamma-v) u\left(x_{t s}\right) .
$$

Now, we claim that, with $k$ defined in (2.3), there is $C>0$ s.t.

$$
\begin{aligned}
g(H) u\left(x_{t s}\right) \gamma u\left(x_{t s}\right) g(H) \leq & k g(H) u\left(x_{t s}\right)^{2} g(H) \\
& +C s^{-1} g(H) \tilde{u}\left(x_{t s}\right)^{2} g(H)
\end{aligned}
$$

where $\tilde{u}\left(x_{t s}\right)^{2}$ is an admissible function. To see this, we first estimate

$$
\begin{aligned}
\mid\left\langle\psi, g(H) u\left(x_{t s}\right)\right. & \left.\gamma u\left(x_{t s}\right) g(H) \psi\right\rangle \mid \\
& \leq\left\|\nabla\langle x\rangle u\left(x_{t s}\right) g(H) \psi\right\|\left\|p u\left(x_{t s}\right) g(H) \psi\right\| .
\end{aligned}
$$

This inequality, together with (4.1), gives

$$
\begin{aligned}
& \left|\left\langle\psi, g(H) u\left(x_{t s}\right) \gamma u\left(x_{t s}\right) g(H) \psi\right\rangle\right| \\
& \quad \dot{\leq}\left\|u\left(x_{t s}\right) g(H) \psi\right\|\left(k\left\|u\left(x_{t s}\right) g(H) \psi\right\|+s^{-1}\left\|\tilde{u}\left(x_{t s}\right) g(H) \psi\right\|\right),
\end{aligned}
$$

which implies (4.8). Now, using (4.8), together with (4.7) and the definitions $u\left(x_{t s}\right)^{2}=f^{\prime}\left(x_{t s}\right)$ and $h\left(x_{t s}\right):=\tilde{u}\left(x_{t s}\right)^{2}$, we obtain

$$
\begin{aligned}
g(H) & D \Phi_{s}(t) g(H) \\
& \dot{\leq}(k-v) s^{-1} g(H) f^{\prime}\left(x_{t s}\right) g(H)+C s^{-2} g(H) h\left(x_{t s}\right) g(H) .
\end{aligned}
$$

Taking the matrix element of this inequality with the vector $\tilde{\psi}_{t}=e^{-i H t} \chi_{b}^{-} \phi$ and using

$$
g(H) \tilde{\psi}_{t}=e^{-i H t} g(H) \chi_{b}^{-} \phi=: \psi_{t},
$$

we find that

$$
\left\langle D \Phi_{s}(t)\right\rangle_{t} \dot{\leq}(k-v) s^{-1}\left\langle f^{\prime}\left(x_{t s}\right)\right\rangle_{t}+C s^{-2}\left\langle h\left(x_{t s}\right)\right\rangle_{t} .
$$


Hence, Eqs. (2.8) and (2.9) and the definition $\Phi_{t s}:=f\left(x_{t s}\right)$ give

$$
\begin{aligned}
\left\langle f\left(x_{t s}\right)\right\rangle_{t}+(v-k) s^{-1} & \int_{0}^{t}\left\langle f^{\prime}\left(x_{r s}\right)\right\rangle_{r} d r \\
& \dot{\leq}\left\langle f\left(x_{0 s}\right)\right\rangle_{0}+C s^{-2} \int_{0}^{t}\left\langle h\left(x_{r s}\right)\right\rangle_{r} d r .
\end{aligned}
$$

Since $k<v$ and since $h=\tilde{u}^{2}$ is an admissible function and therefore $h \gtrsim j^{\prime}$ for some $j \in \mathcal{F}$, (4.13) implies that

$$
\int_{0}^{t}\left\langle f^{\prime}\left(x_{r s}\right)\right\rangle_{r} d r \dot{\lesssim}\left\langle f\left(x_{0 s}\right)\right\rangle_{0}+s^{-1} \int_{0}^{t}\left\langle j^{\prime}\left(x_{r s}\right)\right\rangle_{r} d r
$$

(The constant entering the relation $\lesssim$ here is bounded by a power of $(v-k)^{-1}$.) This, together with the boundedness of $j^{\prime}$, gives estimate (4.3) with $n=0$. Now, assuming (4.3) holds for some $n=n^{\prime} \geq 0$, and using this (with $f=j$ ) for the integral on the r.h.s. of (4.14), we see that (4.3) holds for $n=n^{\prime}+1$.

End of the proof of Theorem 2.1. Recall that $\operatorname{supp} f \subset \mathbb{R}^{+}$for any $f \in \mathcal{F}$, and therefore supp $f\left(x_{0 s}\right) \subset\{\langle x\rangle>a+\delta s\}$. Since $\operatorname{supp} \chi_{b}^{-} \subset\{\langle x\rangle \leq b\}$ and $b<a$, the functions $f\left(x_{0 s}\right)$ and $\chi_{b}^{-}$have disjoint supports. Hence, we have by (A.3) of Lemma A.1 (and the fact that $f^{(k)}\left(x_{0 s}\right) \chi_{b}^{-}=0$ ) that, for any $n$,

$$
f\left(x_{0 s}\right) g(H) \chi_{b}^{-}=\left[f\left(x_{0 s}\right), g(H)\right] \chi_{b}^{-}=O\left(s^{-n}\right)
$$

and therefore, for any $n$ and any $f \in \mathcal{F}$,

$$
\left\langle f\left(x_{0 s}\right)\right\rangle_{0}=\left\langle g(H) \chi_{b}^{-} \phi, f\left(x_{0 s}\right) g(H) \chi_{b}^{-} \phi\right\rangle=O\left(s^{-n}\right) .
$$

Next, retaining the first term in (4.13) and dropping the second one and using (4.3) and (4.16), we conclude that, for any $n$,

$$
\left\langle f\left(x_{t s}\right)\right\rangle_{t} \lesssim s^{-n}
$$

Now, for any $f \in \mathcal{F}$, we have $f(\lambda)=1$ for $\lambda \geq c-v$, and therefore $f\left(x_{t s}\right)=1$ on $\{\langle x\rangle \geq a+v t+(c-v) s\}$. Recalling that $s \geq t$ and $A_{\rho}^{ \pm}:=\left\{x \in \mathbb{R}^{d}: \pm\langle x\rangle \geq \pm \rho\right\}$, we have

$$
A_{c t+a}^{+} \subset\left\{f\left(x_{t t}\right)=1\right\} .
$$

Hence, we conclude that $\left\langle\chi_{A_{c t+a}^{+}}\right\rangle_{t} \dot{\lesssim} t^{-n}$. Since $\psi_{t}:=e^{-i H t} g(H) \chi_{b}^{-} \phi$ for any $\phi \in$ $L^{2},\|\phi\|=1$, this implies (2.4).

Proof of Eq. (2.5). By our assumption, $\rho>a+c t$. We set $s=(\rho-a) / c \geq t$. This gives $\rho=a+c s \geq a+c t$ and therefore $A_{\rho}^{+} \subset\{\langle x\rangle \geq a+v t+(c-v) s\} \subset$ $\left\{f\left(x_{t s}\right)=1\right\}$. Using this together with estimate (4.17) in the time-reversed form $\left\|f\left(x_{t s}\right)^{1 / 2} e^{i H t} g(H) \chi_{A_{b}^{-}}\right\| \lesssim s^{-n}$, and the definitions $s=(\rho-a) / c$ and $\alpha_{t}(B):=$ $e^{i H t} B e^{-i H t}$, we arrive at (2.5). 


\section{Proof of Theorem 3.3}

We follow the proof of Theorem 2.1 . We use the notation $A \dot{\leq} B$ and $A \lesssim B$ introduced in the previous section. Let

$$
\psi_{t}:=U_{t} g_{+}(H) \phi_{0}, \quad \phi_{0}:=\chi_{b}^{-} \phi .
$$

We begin by using (2.8) as before and observe that the identities (4.7) and (4.11) still hold for $H_{t}$. However, the relation (4.12) (the trivial pull-through formula) fails. Now, instead of (4.12), we use the pull-through relation (3.6) to pull in $g(H)$ from $\psi_{t}$. For convenience denote $R:=\psi_{t}-g(H) U_{t} \phi_{0}=O\left(t^{-\mu}\right) \phi_{0}$. Then

$$
\begin{aligned}
& \partial_{t}\left\langle\Phi_{t s}\right\rangle_{t}=\left\langle g(H) U_{t} \phi_{0}, D \Phi_{s}(t) g(H) U_{t} \phi_{0}\right\rangle \\
& +\left\langle R, D \Phi_{s}(t) g(H) U_{t} \phi_{0}\right\rangle+\left\langle g(H) U_{t} \phi_{0}, D \Phi_{s}(t) R\right\rangle+\left\langle R, D \Phi_{s}(t) R\right\rangle .
\end{aligned}
$$

We claim that the second line above is $O\left(s^{-1} t^{-\mu}\right)$. This follows from (4.7) and (4.1) for the first two terms; for the third one we can get the better bound $O\left(s^{-1} t^{-2 \mu}\right)$ using

$$
\left\|p\left(U_{t} g_{+}(H)-g(H) U_{t}\right)\right\|=O\left(t^{-\mu}\right),
$$

which follows from (3.5) and estimates (A.18) and (A.20) of Appendix A,

Going back to (5.1), and using also (4.11) to bound the first terms on the r.h.s., we get, for some admissible function $\tilde{f}$,

$$
\begin{aligned}
\partial_{t}\left\langle\Phi_{t s}\right\rangle_{t} & =\left\langle g(H) U_{t} \phi_{0}, D \Phi_{s}(t) g(H) U_{t} \phi_{0}\right\rangle+O\left(s^{-1} t^{-\mu}\right) \\
& \dot{\leq}(k-v) s^{-1}\left\langle g(H) U_{t} \phi_{0}, f^{\prime}\left(x_{t s}\right) g(H) U_{t} \phi_{0}\right\rangle \\
& +C s^{-2}\left\langle g(H) U_{t} \phi_{0}, \tilde{f}\left(x_{t s}\right) g(H) U_{t} \phi_{0}\right\rangle+O\left(s^{-1} t^{-\mu}\right) .
\end{aligned}
$$

Next, passing back to $\psi_{t}$ by using the pull-through relations in the opposite direction and the fact that $\tilde{f}$ is bounded, we obtain

$$
\partial_{t}\left\langle\Phi_{t s}\right\rangle_{t} \leq(k-v) s^{-1}\left\langle f^{\prime}\left(x_{t s}\right)\right\rangle_{t}+C s^{-2}+C s^{-1} t^{-\mu} .
$$

Since $v>k$, we can drop the first term on the r.h.s. and use the basic equality (2.9), the definition $\Phi_{t s}:=f\left(x_{t s}\right)$ and the conditions $\mu>1, s \geq t$, to find

$$
\left\langle f\left(x_{t s}\right)\right\rangle_{t} \leq\left\langle f\left(x_{0 s}\right)\right\rangle_{0}+C s^{-1} \text {. }
$$

For the first term on the r.h.s., we claim

$$
\left\langle f\left(x_{0 s}\right)\right\rangle_{0}=O\left(s^{-2 \mu /(\mu+1)}\right) .
$$

To prove this estimate, we recall $\psi_{t}:=U_{t} g_{+}(H) \chi_{b}^{-} \phi$, note that $\left\langle f\left(x_{0 s}\right)\right\rangle_{0}=$ $\left\|\chi\left(x_{0 s}\right) \psi_{0}\right\|^{2}$, with $\chi^{2}=f$, and pass from $g_{+}(H)$ to $g_{s^{\beta}}(H):=U_{s^{\beta}}^{-1} g(H) U_{s^{\beta}}$, with $\beta<1$, paying with the error $O\left(s^{-\beta \mu}\right)$ :

$$
\chi\left(x_{0 s}\right) \psi_{0}=\chi\left(x_{0 s}\right) g_{+}(H) \chi_{b}^{-} \phi=\chi\left(x_{0 s}\right) g_{s^{\beta}}(H) \chi_{b}^{-} \phi+O\left(s^{-\beta \mu}\right) .
$$

In Lemma A.3 of Appendix A we show that

$$
\chi\left(x_{0 s}\right) g_{s^{\beta}}(H) \chi_{b}^{-}=O\left(s^{\beta-1}\right) .
$$


This, together with the previous estimate and the choice $\beta=1 /(\mu+1)$, yields (after squaring up) (5.5) . (5.5) and (5.4) imply

$$
\left\langle f\left(x_{t s}\right)\right\rangle_{t} \leq C s^{-1}
$$

which, in view of the definition of $f$, gives, after setting $s=t$, Theorem 3.3.

Acknowledgement. We are grateful to anonymous referees for useful remarks and suggestions. The work on this paper was supported in part by NSERC Grant No. NA7901 (JA and IMS), by a start-up grant from the University of Toronto and NSERC Grant No. 06487 (JA and FP) and by NSF grants DMS-1600749 and NSFC11671163 (AS). None of the authors has a conflict of interest.

\section{Appendix A. Commutator expansions}

In this appendix, we present commutator expansions and estimates, first derived in [19] and then improved in [21, 12, 14] (see [4, 8, 15] for the original work). We follow [12] and refer to this paper for details and references. Here, we mention only that, by the Helffer-Sjöstrand formula, a function $f$ of a self-adjoint operator $A$ can be written as

$$
f(A)=\int d \widetilde{f}(z)(z-A)^{-1}
$$

where $\tilde{f}(z)$ is an almost analytic extension of $f$ to $\mathbb{C}$ supported in a complex neighbourhood of $\operatorname{supp} f$. For $f \in C^{n+2}(\mathbb{R})$, we can choose $\tilde{f}$ satisfying the estimates ( see (B.8) of [12]):

$$
\int|d \tilde{f}(z)||\operatorname{Im}(z)|^{-p-1} \lesssim \sum_{k=0}^{n+2}\left\|f^{(k)}\right\|_{k-p-1},
$$

where $\|f\|_{m}:=\int\langle x\rangle^{m}|f(x)|$. Note that [12] requires $f \in C_{0}^{\infty}(\mathbb{R})$, but one can easily extend representation (A.1) and the needed results to $f$ 's satisfying

$$
\sum_{k=0}^{n+2}\left\|f^{(k)}\right\|_{k-2}<\infty
$$

for some $n \geq 1$, which covers the case $f \in C^{\infty}(\mathbb{R})$ with $f^{\prime} \in C_{0}^{\infty}(\mathbb{R})$ which comes up in this work.

The essential commutator estimates are incorporated in the following lemma:

Lemma A.1. Let $f \in C^{\infty}(\mathbb{R})$ be bounded, with $\sum_{k=0}^{n+2}\left\|f^{(k)}\right\|_{k-2}<\infty$, for some $n \geq 1$. Let $x_{s}=s^{-1}(\langle x\rangle-a)$ for $a>0$ and $1 \leq s<\infty$. Suppose that $H$ satisfies (2.1) and let $g \in C_{0}^{\infty}(\mathbb{R})$. Then, for any $n \geq 1$,

$$
\left[g(H), f\left(x_{s}\right)\right]=\sum_{k=1}^{n-1} \frac{s^{-k}}{k !} B_{k} f^{(k)}\left(x_{s}\right)+O\left(s^{-n}\right),
$$


uniformly in $a \in \mathbb{R}$, where $H^{j} B_{k}, j=0,1, k=1, \ldots, n-1$, are bounded operators and $\left\|H^{j} O\left(s^{-n}\right)\right\| \lesssim s^{-n}, j=0,1$. For $n=1$, the sum on the r.h.s. is omitted.

Proof. Omitting the argument $x_{s}$ in $f$ and $f^{(k)}$ and using (B.14)-(B.15) of [12], we have

$$
\begin{aligned}
{[g(H), f] } & =\sum_{k=1}^{n-1} \frac{s^{-k}}{k !} B_{k} f^{(k)}+s^{-n} \operatorname{Re}(s) \\
B_{k} & =a d_{\langle x\rangle}^{k} g(H) \\
\operatorname{Re}(s) & =\int d \widetilde{f}(z)\left(z-x_{s}\right)^{-1} B_{n}\left(z-x_{s}\right)^{-n},
\end{aligned}
$$

where for $n=1$, the sum on the r.h.s. is omitted. Now we show that the operators $H^{j} B_{k}, j=0,1, k=1, \ldots, n$, and $\operatorname{Re}(s)$ satisfy the estimates:

$$
\left\|H^{j} B_{k}\right\| \lesssim 1, j=0,1, k=1, \ldots, n, \quad\left\|H^{j} R e(s)\right\| \lesssim 1
$$

uniformly in $a$ (and of course $s$ ). Let $R(z):=(z-H)^{-1}$. We claim that

$$
B_{k}=\sum_{\pi \in \Pi_{k}} \int d \widetilde{g}(z) R(z) C_{k_{1}} \ldots R(z) C_{k_{s}} R(z)
$$

where $\pi=\left(k_{1}, \ldots, k_{s}\right)$, with $1 \leq k_{j} \leq 2$ and $k_{1}+\cdots+k_{s}=k$, are ordered partitions of $k$, and $C_{j}:=a d_{\langle x\rangle}^{j} H$. Indeed, using the formula (A.1) for $g(H)$,

$$
g(H)=\int d \widetilde{g}(z) R(z)
$$

and the relation $a d_{\langle x\rangle} R=R C_{1} R$, we see that this is true for $k=1$ :

$$
B_{1}=\int d \widetilde{g}(z) R(z) C_{1} R(z)
$$


Now, assuming that (A.8) holds for $k=m$, we prove it for $k=m+1$. To this end, we use that $a d_{\langle x\rangle} B_{j}=B_{j+1}, a d_{\langle x\rangle} R=R C_{1} R$ and $a d_{\langle x\rangle} C_{j}=C_{j+1}$ to obtain

$$
\begin{aligned}
B_{m+1}=\sum_{\pi \in \Pi_{m}} & \int d \widetilde{g}(z)\left\{\sum_{a=1}^{m} \prod_{j=1}^{a-1}\left(R(z) C_{k_{j}}\right)\left(R(z) C_{1} R(z) C_{k_{a}}+R(z) C_{k_{a}+1}\right)\right. \\
& \left.\times \prod_{j=a+1}^{s}\left(R(z) C_{k_{j}}\right) R(z)+\prod_{j=1}^{s}\left(R(z) C_{k_{j}}\right)\left(R(z) C_{1} R(z)\right)\right\} \\
= & \sum_{\pi \in \Pi_{m}} \int d \widetilde{g}(z)\left\{\sum _ { a = 1 } ^ { m } \left[\prod_{j=1}^{a-1}\left(R(z) C_{k_{j}}\right)\left(R(z) C_{1}\right) \prod_{j=a}^{s}\left(R(z) C_{k_{j}}\right)\right.\right. \\
& \left.+\prod_{j=1}^{a-1}\left(R(z) C_{k_{j}}\right)\left(R(z) C_{k_{a}+1}\right) \prod_{j=a+1}^{s}\left(R(z) C_{k_{j}}\right)\right] \\
& \left.+\prod_{j=1}^{s}\left(R(z) C_{k_{j}}\right)\left(R(z) C_{1}\right)\right\} R(z) .
\end{aligned}
$$

It is not hard to see that (A.11) is of the form (A.8) with $k=m+1$. Finally, since $a d_{\langle x\rangle}^{k} H=0$, for $k \geq 3$, we have that either $k_{a}+1 \leq 2$ or $C_{k_{a}+1}=0$.

To prove boundedness of $B_{k}$, we use that $C_{1}:=[\langle x\rangle, H]=i \gamma$, where, recall, $\gamma=\frac{1}{2}(p \cdot \nabla\langle x\rangle+\nabla\langle x\rangle \cdot p)$, with $p:=-i \nabla$, and $C_{2}:=[\langle x\rangle,[\langle x\rangle, H]]=-|\nabla\langle x\rangle|^{2}$. Let

$$
S:=(H+c)^{1 / 2}, \text { where } c:=-\inf H+1,
$$

and $(H+c)^{s}, s \in \mathbb{R}$, is defined by the spectral theory. Since $\gamma$ is $S$-bounded, we have $\|\gamma R(z)\| \lesssim\|S R(z)\| \lesssim \sup _{s \geq 1}\left(s^{1 / 2}|s-c-z|^{-1}\right)$, which gives

$$
\left\|C_{1} R(z)\right\| \lesssim\langle\operatorname{Re} z\rangle^{1 / 2} /|\operatorname{Im} z|
$$

Using this and $|\nabla\langle x\rangle| \leq 1$ in (A.8) and using (A.2) for $g$ and the fact that $\widetilde{g}(z)$ supported in a complex neighbourhood of supp $g$, which is a compact set, shows that $H^{j} B_{k}, k=1,2, \ldots, j=0,1$, are bounded.

The proof of the last estimate in (A.7) is similar (cf. (B.8) and also (B.15) of [12]).

Now, we prove various commutator estimates used in the main text.

\section{Lemma A.2.}

$$
\left[g(H), W_{r}\right]=O\left(r^{-\mu-1}\right) .
$$

Proof. As in (A.4), with $n=1$, we have

$$
\left[W_{r}, g(H)\right]=\int d \widetilde{g}(z)(z-H)^{-1}\left[W_{r}, H\right](z-H)^{-1}
$$


Using this, estimate (A.2) for $\widetilde{g}(z)$ and the fact that $\left[W_{r}, H\right]=\nabla W_{r} \cdot \nabla+\frac{1}{2} \Delta W_{r}$ is $S$-bounded, where the operator $S$ is given in (A.12), and the estimate

$$
\|p R(z)\| \lesssim|\operatorname{Re} z|^{1 / 2} /|\operatorname{Im} z|
$$

(cf. (A.13) ), we arrive at (A.14).

Lemma A.3. Estimate (5.6)), that is, $\left\|\chi\left(x_{0 s}\right) g_{s^{\beta}}(H) \chi_{b}^{-}\right\|=O\left(s^{\beta-1}\right)$, holds.

Proof. Recall the definition $g_{s^{\beta}}(H):=U_{s^{\beta}}^{-1} g(H) U_{s^{\beta}}$ and let $\chi \equiv \chi\left(x_{0 s}\right)$. Using $\chi \chi_{b}^{-}=0$, we write

$$
\begin{aligned}
\chi g_{s^{\beta}}(H) \chi_{b}^{-}=\left[\chi, U_{s^{\beta}}^{-1}\right] g(H) U_{s^{\beta}} \chi_{b}^{-}+U_{s^{\beta}}^{-1}[\chi, g(H)] U_{s^{\beta}} \chi_{b}^{-} \\
+U_{s^{\beta}}^{-1} g(H)\left[\chi, U_{s^{\beta}}\right] \chi_{b}^{-} .
\end{aligned}
$$

Since $\left[\chi, U_{s^{\beta}}\right]=U_{s^{\beta}}\left(U_{s^{\beta}}^{-1} \chi U_{s^{\beta}}-\chi\right)=U_{s^{\beta}} \int_{0}^{s^{\beta}} \partial_{r}\left(U_{r}^{-1} \chi U_{r}\right) d r$ and $\partial_{r}\left(U_{r}^{-1} \chi U_{r}\right)=$ $i U_{r}^{-1}\left[H_{r}, \chi\right] U_{r}$, we have

$$
\left[\chi, U_{s^{\beta}}\right]=i U_{s^{\beta}} \int_{0}^{s^{\beta}} U_{r}^{-1}\left[H_{r}, \chi\right] U_{r} d r .
$$

Note that $\left[H_{r}, \chi\right]=-i p \nabla \chi+\frac{1}{2}(\Delta \chi)$. We control $p$ by $S^{-1}=(H+c)^{-1 / 2}$ (see (A.12) ) as

$$
p=S B=B^{\prime} S,
$$

where $B:=(H+c)^{-1 / 2} p$ and $B^{\prime}:=p(H+c)^{-1 / 2}$, bounded operators. Eq. (A.17), together with the last two relations, gives

$$
\left[\chi, U_{s^{\beta}}\right]=U_{s^{\beta}} \int_{0}^{s^{\beta}} U_{r}^{-1}\left(S B \nabla \chi+i \frac{1}{2}(\Delta \chi)\right) U_{r} d r .
$$

Next, we commute $(H+c)^{1 / 2}$ to the left. To this end, we apply the equation

$$
\left[S, U_{r}\right]=O(1)
$$

which we now prove. First, we write $S=(H+c)^{1 / 2}=(H+c)(H+c)^{-1 / 2}$ and use the explicit formula $(H+c)^{-s}:=c^{\prime} \int_{0}^{\infty}(H+c+\omega)^{-1} d \omega / \omega^{s}$, where $s \in(0,1)$ and $c^{\prime}:=\left[\int_{0}^{\infty}(1+\omega)^{-1} d \omega / \omega^{s}\right]^{-1}$, to obtain $\left[W_{r},(H+c)^{1 / 2}\right]=O\left(r^{-\mu-1}\right)$. This implies the estimate $\left[H_{r},(H+c)^{1 / 2}\right]=\left[W_{r},(H+c)^{1 / 2}\right]=O\left(r^{-\mu-1}\right)$, which, together with $\mu>0$ and the relation

$$
\left[S, U_{r}\right]=U_{r} \int_{0}^{r} i U_{r^{\prime}}^{-1}\left[H_{r^{\prime}}, S\right] U_{r^{\prime}} d r^{\prime},
$$

yields (A.20).

Commuting $S$ in Eq. (A.19) to the left (either twice through $U_{r}^{-1}$ and $U_{s^{\beta}}$, or once through $\left.U_{s^{\beta}} U_{r}^{-1}=U\left(s^{\beta}, r\right)\right)$ and using (A.20), $\nabla \chi=O\left(s^{-1}\right)$ and $\Delta \chi=O\left(s^{-2}\right)$, 
gives

$$
\begin{aligned}
{\left[\chi, U_{s^{\beta}}\right] } & =S O\left(s^{\beta-1}\right)+\int_{0}^{s^{\beta}}\left(O\left(s^{-1}\right)+O\left(s^{-2}\right)\right) U_{r} d r \\
& =S O\left(s^{\beta-1}\right)+O\left(s^{\beta-1}\right) .
\end{aligned}
$$

A similar estimate holds for $\left[\chi, U_{s^{\beta}}^{-1}\right]=-\left[\chi, U_{s^{\beta}}\right]^{*}$ :

$$
\left[\chi, U_{s^{\beta}}^{-1}\right]=O\left(s^{\beta-1}\right) S+O\left(s^{\beta-1}\right) .
$$

Now, the second term on the r.h.s. of the above relation produces the right bound, $O\left(s^{-1+\beta}\right)$ and so does the first term multiplied by $g(H)$, as $(H+1)^{1 / 2} g(H)$ is a bounded operator. This shows that the first term on the r.h.s. of (A.16) is of the order $O\left(s^{-1+\beta}\right)$. The same estimates apply to the third term on the r.h.s. of (A.16) giving $O\left(s^{-1+\beta}\right)$. For the second term on the r.h.s. of (A.16), we use (A.3) to obtain $[\chi, g(H)]=O\left(s^{-1}\right)$. This proves (5.6).

\section{REFERENCES}

[1] J.-F. Bony, J. Faupin, I.M. Sigal : Maximal velocity of photons in nonrelativistic QED. Adv. Math. 231, 3054-3078 (2012).

[2] H. Cycon, R. Froese, W. Kirsch and B. Simon : Schrödinger operators. Texts and Monographs in Physics, Springer Verlag (1987).

[3] J. Dereziński and C. Gérard : Scattering Theory of Classical and Quantum N-Particle Systems. Springer-Verlag: Berlin, 1997.

[4] E.B. Davies: Spectral Theory and Differential Operators. Cambridge University Press (1995).

[5] D. V. Else, F. Machado, Ch. Nayak, and N. Y. Yao : Improved Lieb-Robinson bound for many-body Hamiltonians with power-law interactions. Phys. Rev. A 101, 022333, 2020.

[6] V. Enss: Propagation properties of quantum scattering states. J. Funct. Anal. 52 (1983), no. 2, 219-251.

[7] J. Faupin, I.M.Sigal : On Rayleigh scattering in non-relativistic quantum electrodynamics. Commun. Math. Phys. 328, 1199-1254 (2014).

[8] B. Helffer and J. Sjöstrand : Equation de Schrödinger avec champ magnétique et équation de Harper. In : Schrödinger operators. H. Holden, A. Jensen eds., Lecture Notes in Physics Vol. 345 Springer Verlag (1989).

[9] M. Gebert, B. Nachtergaele, J. Reschke, R. Sims: Lieb-Robinson bounds and strongly continuous dynamics for a class of many-body fermion systems in $\mathbb{R}^{d}$. Annales Henri Poincaré : 2020.

[10] I. Herbst and E. Skibsted : Free channel Fourier transform in the long-range N-body problem. J. d'Analyse Math. 65 (1995) 297-332.

[11] Shanlin Huang and A. Soffer, Uncertainty principle, minimal escape velocities and observability inequalities for Schrödinger equations, Amer. Math Journal (to appear), arXiv 
[12] W. Hunziker and I.M. Sigal : Time-dependent scattering theory of n-body quantum systems, Reviews in Mathematical Physics, Vol. 12, No. 8 (2000) 1033-1084.

[13] W. Hunziker and I.M. Sigal : The quantum N-body problem, J. Math. Phys. 2000.

[14] W. Hunziker, I.M. Sigal and A. Soffer: Minimal escape velocities, Comm. PDEs.

[15] V. Ivrii and I.M. Sigal, Asymptotics of the ground state energies of large Coulomb systems, Annals of Math. 138 (1993) 243-335.

[16] T. Matsuta, T. Koma \& S. Nakamura: Improving the Lieb-Robinson Bound for Long-Range Interactions. Annales Henri Poincaré volume 18, 519-528 (2017)

[17] B. Nachtergaele and R. Sims: Much ado about something: why Lieb-Robinson bounds are useful. arXiv:1102.0835.

[18] I.M. Sigal : On long range scattering. Duke Math. J. 60 (1990) 473-496.

[19] I.M. Sigal and A. Soffer : Local decay and propagation estimates for timedependent and time-independent Hamiltonians. Preprint, Princeton Univ. (1988). http://www.math.toronto.edu/sigal/publications/SigSofVelBnd.pdf.

[20] I.M. Sigal and A. Soffer : Long-range many-body scattering. Invent. Math. 99 (1990) 115-143.

[21] E. Skibsted : Propagation estimates for N-body Schrödinger operators. Comm. Math. Phys. 142 (1992) 67-98.

Jack Arbunich, Department of Mathematics, University of Toronto, 40 St. George street, Toronto, M5S 2E4, Ontario, Canada

Email address: jack.arbunich@utoronto.ca

Fabio Pusateri, Department of Mathematics, University of Toronto, 40 St. George Street, Toronto, M5S 2E4, Ontario, Canada

Email address: fabiop@math.toronto.edu

Israel Michael Sigal, Department of Mathematics, University of Toronto, 40 St. George street, Toronto, M5S 2E4, Ontario, Canada

Email address: im.sigal@utoronto.ca

Avy Soffer, Mathematics Department, Rutgers University, New Brunswick, NJ 08903, USA

Email address: soffer@math.rutgers.edu 